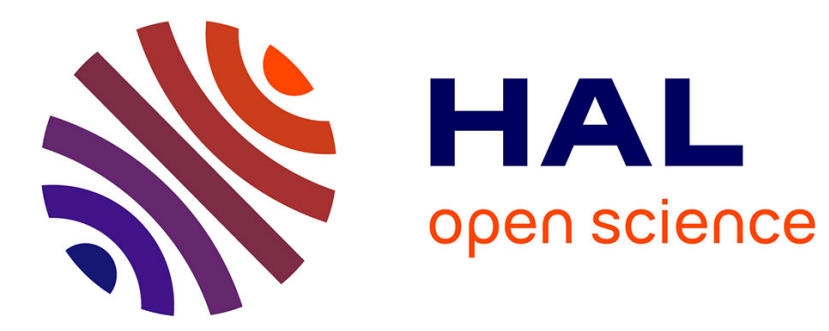

\title{
Tape casting of proton conducting ceramic material
}

Rémi Costa, Julien Hafsaoui, Ana Paula Almeida de Oliveira, Arnaud

Grosjean, Matthieu Caruel, Anthony Chesnaud, Alain Thorel

\section{To cite this version:}

Rémi Costa, Julien Hafsaoui, Ana Paula Almeida de Oliveira, Arnaud Grosjean, Matthieu Caruel, et al. Tape casting of proton conducting ceramic material. Journal of Applied Electrochemistry, 2009, 39, pp.485-495. 10.1007/s10800-008-9671-7 . hal-00379097

\section{HAL Id: hal-00379097 https://hal.science/hal-00379097}

Submitted on 25 Jun 2013

HAL is a multi-disciplinary open access archive for the deposit and dissemination of scientific research documents, whether they are published or not. The documents may come from teaching and research institutions in France or abroad, or from public or private research centers.
L'archive ouverte pluridisciplinaire HAL, est destinée au dépôt et à la diffusion de documents scientifiques de niveau recherche, publiés ou non, émanant des établissements d'enseignement et de recherche français ou étrangers, des laboratoires publics ou privés. 


\title{
Tape Casting of Proton Conducting Ceramic Material
}

\author{
Rémi Costa, Julien Hafsaoui, Ana Paula Almeida De Oliveira, Arnaud Grosjean, \\ Matthieu Caruel, Anthony Chesnaud, Alain Thorel* \\ Centre des Matériaux Pierre-Marie Fourt, UMR CNRS 7633, Mines ParisTech, BP 87, 91003 \\ Evry Cedex (France) \\ Centre Énergétique et Procédés, Mines ParisTech, 60 boulevard Saint-Michel, 75006 Paris \\ (France)
}

\author{
* Corresponding Author: \\ Phone: +330160763030 \\ Fax: +330160763150 \\ e-mail: $\underline{\text { alain.thorel@ensmp.fr }}$
}

\begin{abstract}
This work explores experimental procedures for tape-cast proton conducting ceramic fuel cells (PCFC) based on Yttrium-doped Barium Cerate (BCY10). The work is based on several years experience on aqueous tape-casting applied to the shaping of YSZ-based SOFC: however, water-based tape casting of BCY10 appeared to be impracticable for reasons associated with the high basicity of this material that results in rapid hydrolysis when in contact with water. Organic tape casting was therefore developed for BCY10, but only on Electrolyte (BCY10)/Anode (BCY10 + NiO) half cells since up to now no cathode material is available. Planar $20 \mathrm{~mm}$ diameter circular half-cells were obtained with the aid of a small load on top of the bi-layer to counterbalance the inevitable warping of the samples. Back-scattered SEM and X-Ray computer-controlled microtomography showed sedimentation of some large grains in the green tapes which are believed to have formed by a mechanism associated with a porosity gradient. We have modelled the deformation that occurs during sintering taking into account the elastic, thermal, viscoplastic, and sintering components of the total deformation. 2D and 3D Finite Element numerical simulations showed that the driving force for the deformation is associated with this porosity gradient.
\end{abstract}

Keywords: PCFC, protonic conductor, tape-casting, sintering, microtomography.

\section{Introduction}

Solid Oxide Fuel Cells (SOFC) are promising power generating systems which are currently based on anionic solid conductors, mostly Yttria-Stabilized Zirconia (YSZ). The operating 
temperature of YSZ-based SOFC is usually high $\left(>900^{\circ} \mathrm{C}\right)$ in order to reach the highest anionic conduction performance. Operating SOFC at such an elevated temperature gives significant advantages (chemical stability with $\mathrm{CO}$ and $\mathrm{CO}_{2}$, direct $\mathrm{CH}_{4}$ feeding, etc...), but also has severe drawbacks associated with mechanical failure of components and chemical instabilities during ageing and cycling. These difficulties can be significantly diminished if the operating temperature is reduced to $600{ }^{\circ} \mathrm{C}-700{ }^{\circ} \mathrm{C}$. To compensate for the loss of conductivity associated with the temperature drop, two main avenues have been considered: enhance the oxygen ion conductivity by increasing the ion mobility by heterovalent doping with oxygen conductors such as Ceria, and/or by decreasing the electrolyte ohmic loss by reducing its thickness. However, another challenging alternative has been recently proposed in which the classical mechanism of oxygen conduction is replaced by the conduction of protons $\left(\mathrm{H}^{+}\right)$. Though materials exhibiting protonic conduction - mostly perovskites - have been known for at least two decades, it is only recently that they have been considered for the electrolyte in Proton Conduction Fuel Cells (PCFC). Among all the perovskite based proton conductors, $10 \%$ Yttria-substituted $\mathrm{BaCeO}_{3}$ (BCY10) is widely studied for its high level of protonic conduction below $700{ }^{\circ} \mathrm{C}$ under hydrogen-and/or water-containing atmospheres $\left(10^{-2}\right.$ $\mathrm{S} \mathrm{cm}^{-1}$ at $600{ }^{\circ} \mathrm{C}$ under wet hydrogen). Based on our experience on the shaping of classical SOFC, we propose in the present work to apply and adapt the tape casting techniques to the case of PCFC.

\section{General considerations on tape casting and preliminary work}

\subsection{General considerations on tape casting.}

Tape casting is a well-known low cost process for ceramic material shaping, particularly wellsuited for the fabrication of thin $(20 \mu \mathrm{m}$ up to $500 \mu \mathrm{m})$ flat components. It can be used to produce a wide variety of controlled morphologies, from highly porous to fully dense microstructures. Starting powders of different natures can be incorporated and mixed in the slurry, and tape casting offers also the very useful possibility of stacking green tapes to obtain a multilayered final product. For a given powder, the sintering behaviour of green tapes, and hence their final microstructure, depends on the arrangement, dispersion and homogeneity of the starting ceramic powder particles in the slurry [1]. As a consequence, the slurry formulation is the most crucial step in the shaping process. Generally, the slurry is composed of a mixture of several organic and inorganic compounds. The organic components are usually the binder, the dispersant, the plasticizer [2], the solvent in the case of organic tape 
casting, and possibly some other additives like wetting agents, defoamers, or pore formers if porosity is desired in the final microstructure. The inorganic compounds include the ceramic powders to be shaped, sintering additives, and water as the solvent for aqueous tape casting. All the organic components remain in the green tape after drying. Since they are removed when heated at elevated temperature, they give rise to pores, which cannot always be eliminated during the sintering treatment. Consequently, the ratio of the amount of powder and organic compounds, and hence the final formulation of the slurry, must be fine-tuned in order to tailor the final microstructure. Amongst the slurry characteristics, the stability is of utmost importance. Polarization interactions must take place at the solid-liquid interface, interactions whose intensity governs the slurry stability [1]. Consequently, the value of the dielectric constant of the liquid determines the slurry stability, and hence the choice of solvent, which in turn determines the choice of all the other additives. The other forces acting on the particles in the slurry are gravity, which depends on the particles mass (and indirectly size), and the attractive Van der Waals interactions, which promote flocculation and act against the stability of the slurry. On the other hand, thermal agitation, electrostatic and steric repulsive forces promote the dispersion of the particles and therefore increase the stability of the slurry. The role of the dispersant agent is precisely to enhance the intensity of these dispersive forces. The second important slurry characteristic is the viscosity, which determines the viability of the process to cast green tapes. The slurry viscosity varies as a function of the amount of solvent per unit volume; it needs to be precisely adjusted to allow for a good dispersion of the powder as well as for an efficient dissolution of the binder.

\subsection{Preliminary work on the aqueous tape casting of SOFC.}

We have applied routinely water-based tape casting to shape SOFC single cells in a single operation [3]. The starting materials were the following:

- $\quad$ Electrolyte: YSZ (TZ8-Y, Tosoh),

- $\quad$ Anode: porous Ni-YSZ cermet NiO (Ni precursor = NiO, Novamet A Type),

- $\quad$ Cathode: composite YSZ- $\mathrm{La}_{0.80} \mathrm{Sr}_{0.20} \mathrm{MnO}_{3-\delta}$ (LSM, Rhodia).

The slurry composition for each layer was adjusted to obtain the same shrinkage for the three components of the cell; the thickness of the cast tape was adjusted so that the thermomechanical stresses were minimized during the sintering treatment following Timoshenko's bilayer model [4]. The slurries composition was based on the following chemicals: 
- Water/sodium alginate in the ratio (100:1) as the couple solvent/binder,

- $\quad$ Dispersant: Dolapix ET85,

- $\quad$ Macro pore-former when needed: acrylic polymer.

Slurries for the electrodes and for the electrolyte were cast separately, with appropriate thickness, on a glass support. Green tapes were then obtained by a chemical gelation of the alginate through the complexation of $\mathrm{Ca}^{2+}$ ions in the presence of $\mathrm{CaCl}_{2}$ solution. The three different green-tapes were then cut and stacked to obtain a full green single cell. The threelayer stacks obtained in this way were then dried between two hydrophilic honeycomb supports at $70{ }^{\circ} \mathrm{C}$ so that the water could be desorbed symmetrically from the two surfaces to avoid any warping of the stack. The activation energy of the drying process proved to correspond to the extraction of water by capillary forces instead of a mechanism based on the evaporation of water [3]. After the drying was completed, the stacks were placed between two Mullite alveolar supports, whose weight was sufficient to maintain the flatness of the cell, but small enough to allow for the cell shrinkage during the sintering heat treatment (Fig. 1). Twoinch flat SOFC single cells with high quality interfaces between electrolyte and electrodes were routinely obtained (Fig. 2). Green stacks must obviously be sintered at a common temperature, which was determined to be $1350{ }^{\circ} \mathrm{C}$ in our case; unfortunately, this temperature led to a significant level of reactivity between the YSZ and LSM, and hence to the appearance of highly resistive pyrochlore phases at interfaces between the electrolyte and the cathode material, which strongly impaired the anionic conductivity [3,5]. To overcome this problem, we have developed a sequential organic tape casting approach to cast the cathode layer on top of a sintered Anode/Electrolyte half-cell prepared by the process described above. It is to be noted here that, owing to surface tension considerations, water-based tape casting does not lend itself to the successful deposit of a layer on top of an already sintered material. With this method the cathode could be heat-treated at a lower temperature $\left(1200{ }^{\circ} \mathrm{C}\right.$ for 1 hour $)$, well below the temperature where reactivity with YSZ occurs. Consequently, high quality interfaces between cathode and electrolyte could be obtained (Fig. 3), with no detectable electrically resistive phases.

\section{Experimental}

\subsection{General considerations on proton conducting materials.}

$\mathrm{ABO}_{3}$ perovskites based on $\mathrm{BaCeO}_{3}$ or $\mathrm{SrCeO}_{3}$ have demonstrated high proton conduction performances. They are being considered for use as electrolyte materials for Proton 
Conducting Fuel Cells (PCFC). The partial substitution of the B-site cation $\left(\mathrm{Ce}^{4+}\right)$ by an aliovalent cation (such as $\mathrm{Y}^{3+}$ ) creates oxygen vacancies in the oxygen atom sublattice. Due to the difference of electronegativity between cations and anions of the perovskite, the $\mathrm{O}^{2-}$ sublattice exhibits a strong basic behaviour. As a consequence, $\mathrm{O}^{2-}$ anions associated with vacancies react with water vapour to create protonic defects (Equation 1), which are responsible for the protonic conduction.

$$
\mathrm{H}_{2} \mathrm{O}_{(\mathrm{g})}+\mathrm{V}_{\mathrm{O}}^{\ddot{*}}+\mathrm{O}_{\mathrm{O}}^{\mathrm{X}}=2\left(\mathrm{OH}_{\mathrm{O}}^{\cdot}\right)
$$

Among all of these promising materials, $\mathrm{BaCe}_{0.90} \mathrm{Y}_{0.10} \mathrm{O}_{3-\delta}$ (BCY10) has been identified by most researchers as exhibiting one of the highest protonic conductivity at $600{ }^{\circ} \mathrm{C}\left(10^{-2} \mathrm{~S} \mathrm{~cm}^{-1}\right.$ under wet hydrogen). In this study, we propose to use this material as the electrolyte of a PCFC. Since there is no specific cathode material for PCFC available up to now, this work focusses on how tape casting and co-sintering can be used for the fabrication of Anode/Electrolyte half-cells (symmetrical or not). Following what is classically done for SOFC, the anode here is made of a mixture of $\mathrm{Ni}$ and of the electrolyte material, with the three anode components (Ni, BCY10 and porosity) arranged so that the volume fraction of each is above the $3 \mathrm{D}$ percolation threshold.

\subsection{Starting materials and composition of the slurries.}

BCY10 was produced via a chemical route based on oxalate co-precipitation following exactly the procedure described by Almeida de Olivera et al. [6]. The as-calcined powder was identified by X-Ray Diffraction (XRD) as being pure BCY10 (Fig. 4); it exhibits a specific surface area of $2.3 \mathrm{~m}^{2} \mathrm{~g}^{-1}$ (BET measurement), with a very anisotropic grain shape (Fig. 5). For the Anode cermet, a commercial NiO powder (Novamet A type) has been chosen as the Ni precursor. Water-based tape casting was not applicable for BCY10 shaping mainly for two reasons:

- $\quad$ BCY10 exhibits a strong basic behaviour; since water is an amphoteric electrolyte, the $\mathrm{pH}$ of such a suspension is quite high (Fig. 6), and hydrolysis of the material occurs rapidly;

- Tests suggested an adsorption of Alginate molecules at the surface of the powder, which inhibits the possibility of using it to make a gel. 
Among all of the organic solvent/binder couples [2], we have chosen the couple

Ethanol/PVB: it limits the toxicity of the slurries and makes stable BCY10 suspensions rather easily. The following products were used:

- $\quad$ Absolute Ethanol (Fisher Scientific),

- $\quad$ Poly(Vinyl Butyral) (PVB) (Acros Organics) as the binder (and as a pore former in the case of the anode),

- $\quad$ PolyEthylene Glycol-400 grade (PEG) (Fisher Scientific) as the plasticizer,

- $\quad$ Glycerol (Fisher Scientific) as an anti-adhesive.

Table 1 gives the optimized composition for the Anode and Electrolyte slurries in regard to the desired final microstructures (dense BYC10, porous anode with interconnected $\mathrm{Ni}$ particles, BCY10 and network of pores) [7]. Slurries were ball-milled for 4 hours prior to casting in order to obtain an homogeneous suspension.

\begin{tabular}{lll}
\hline TABLE 1. Composition (in g) of optimized slurries in regards to the final microstructure. \\
\hline Chemicals & Anode / g & Electrolyte / g \\
\hline BCY10 & 2.5 & 6.0 \\
$\mathrm{NiO}$ & 2.7 & - \\
Ethanol & 4.5 & 4.8 \\
PVB & 2.0 & 0.60 \\
PEG & 0.70 & 0.30 \\
Glycerol & 0.45 & - \\
\hline
\end{tabular}

\subsection{Experimental procedures for tape casting.}

Slurries were cast on a glass support coated with glycerol to prevent the adhesion of the green tapes. First a $500 \mu \mathrm{m}$ thick anode layer was cast, and left twenty minutes at room temperature for the drying process to start taking place. Then a $150 \mu \mathrm{m}$ thick electrolyte layer was cast on top of the anode tape. For the fabrication of symmetric half-cells, a second $500 \mu \mathrm{m}$ thick anode layer was cast on top of the electrolyte layer, again twenty minutes later. The stack of green tapes was then dried at room temperature for two hours before being cut into discs. $\mathrm{BaCeO}_{3}$ based perovskite reacts at high temperature $\left(>1200{ }^{\circ} \mathrm{C}\right)$ with most of the materials used as sintering supports $\left(\mathrm{Al}_{2} \mathrm{O}_{3}, \mathrm{ZrO}_{2}\right)$ [8-10]. Therefore, the sintering of BCY10 was made on a BCY10 powder layer. Figure 7 shows the optimized sintering thermal treatment [7]. 


\section{Results and Discussion}

In the case of symmetrical half-cells, planar samples of about $20 \mathrm{~mm}$ in diameter were obtained if they were sintered under a small, homogeneous load. The samples were crack free, with a high quality of interface (Fig. 8 and Fig. 9) [7]. In all the other cases, for anode or electrolyte mono- and bi-layers, a strong curvature was systematically observed, with or without a small load. Transmission Electron Microscopy (TEM) investigations (Energy Dispersive X-Ray [EDX] analyses in Scanning Transmission Electron Microscopy [STEM] mode) coupled with XRD revealed the systematic contamination of the BCY10 phase by NiO, and suggest a B-site substitution of Ce atoms by Ni atoms [7]. The impact of the Nicontamination upon the protonic conduction is currently under investigations. Microstructural investigations, carried out by Scanning Electron Microscopy (SEM) and X-Ray computercontrolled Microtomography (ESRF, ID19 beamline, with $34 \mathrm{keV}$ X-Ray energy, and a spatial resolution of $0.56 \mu \mathrm{m}_{\text {pixel }}{ }^{-1}$ ), were carried out to understand the origin of, and cure for, the deformation of the samples during sintering.

\subsection{Characterization of the microstructure.}

Interrupted sintering tests were conducted to follow the evolution of the porosity within the samples during the thermal treatment. The porosity was measured by image analysis on BackScattered-SEM images on polished cross sections. The quantitative porosity measurements gave evidence of the existence of a small porosity gradient in the $\mathrm{z}$ direction of the layers. This porosity gradient was confirmed by X-ray computer-controlled Microtomography investigations on samples for different durations of sintering (Fig. 10 and 11), and was found to be associated with a grain size gradient in the same $\mathrm{z}$ direction; the largest grains are found at the bottom of the layer whereas the smallest are observed close to the opposite surface. These observations suggest that a sedimentation process starts to act from the moment the tapes are cast until the green tapes are completely dried. The gradient of porosity is suspected to be the driving force for the deformation during sintering, the more dispersed the grain size distribution the more severe the deformation. The impact of this small porosity gradient on the curvature of the sintered samples has been approached via a phenomenological sintering model that will be described below.

\subsection{Modelling of the deformation of the tapes during sintering.}

During the sintering process the material is submitted to irreversible deformations that occur at high temperature. These deformations are therefore thermally activated and closely linked 
to the reduction of the porosity. The key-point of the model is to describe how the evolution of porosity influences the irreversible deformation.

The total deformation velocity of a sample during sintering can be written as follows (Equation 2):

$$
\underset{\sim}{\varepsilon}=\underset{\sim}{\varepsilon}+\underset{\sim}{\varepsilon}+\underset{\sim}{\varepsilon}+\underset{\sim}{\varepsilon}
$$

where $\underset{\sim}{\stackrel{\bullet}{\varepsilon}}$ represents the elastic deformation rate; $\underset{\sim}{\stackrel{.}{\varepsilon}}$ th the thermal deformation rate; $\underset{\sim}{\varepsilon}$ the visco-plastic deformation rate and $\dot{\varepsilon}^{s}$ the deformation rate due to the sintering.

\subsubsection{Viscoplasticity deformation and creep.}

In the case of porous materials, the viscoplastic deformation velocity can be described as follows (Equation 3):

$$
\underset{\sim}{\stackrel{v}{\varepsilon}}=(1-f) \times \frac{\partial \Psi}{\partial \Phi} \times \frac{\partial \Phi}{\partial \sigma}
$$

where $\Psi$ is the viscoplastic potential and $\Phi$ the plasticity threshold. To compute Equation 3, an equivalent stress as a function of porosity $\mathrm{f}$ is defined (Equation 4$)$ :

$$
\sigma=\sqrt{\mathrm{C}(\mathrm{f}) \times \sigma_{\text {eq }}^{2}+\mathrm{F}(\mathrm{f}) \times(\operatorname{tr}(|\sigma|))^{2}}
$$

Assuming no irreversible deformation, the direction of the viscoplastic flow is given by (Equation 5):

$$
\Phi=\sigma^{*} \Rightarrow \underset{\sim}{\mathrm{n}}=\frac{\partial \sigma^{*}}{\partial \sigma}
$$

The creep velocity can be described by a thermally activated Norton's law (Equation 6):

$$
\dot{\mathrm{p}}=\frac{\partial \Psi}{\partial \Phi}=\mathrm{K}_{\mathrm{o}} \mathrm{e}^{\frac{-\mathrm{Q}}{\mathrm{RT}}}\left(\sigma^{*}\right)^{\mathrm{n}}
$$

By combining Equation 6 and Equation 7 into Equation 3, the general expression of the viscoplastic deformation velocity is obtained (Equation 7):

$$
\underset{\sim}{\stackrel{\bullet}{\varepsilon}}=(1-f) K_{0} e^{\frac{-Q}{R T}}\left(\sigma^{*}\right)^{n} \frac{\partial \sigma^{*}}{\partial \sigma}
$$




\subsubsection{Shrinkage due to Sintering.}

Sintering is assumed first to be isotropic. As a consequence the sintering deformation velocity is a function of porosity and temperature. Assuming a thermally activated sintering process and limiting the porosity corresponding to the maximal compactness of the material, the sintering deformation velocity can be described by equation (Equation 8):

$$
\underset{\sim}{\stackrel{\bullet}{\varepsilon}}=-\mathrm{A}_{0} \mathrm{e}^{\frac{\mathrm{Q}}{\mathrm{RT}}\left(\mathrm{f}-\mathrm{f}^{\infty}\right)^{\beta}} \underset{\sim}{\mathrm{I}}
$$

The sintering activation energy $\mathrm{Q}$ will be the same as the creep activation energy, assuming that both phenomena are controlled by self-diffusion.

\subsubsection{General equations for the material behaviour.}

The mechanical deformation velocity can be expressed by the summation of a reversible and an irreversible part (Equation 9):

$$
\stackrel{. \mathrm{m}}{\varepsilon}=\underset{\sim}{\varepsilon}+\dot{\sim}_{\tilde{\varepsilon}}^{. \text {irr }}
$$

The elastic deformation velocity can be described by Hooke's law for a homogeneous and isotropic material (Equation 10):

$$
\underset{\sim}{\varepsilon}=\underset{\sim}{\mathrm{C}^{-1}}: \underset{\sim}{\sigma}
$$

The irreversible part consists of the visco-plastic deformation velocity and the sintering deformation velocity (Equation 11):

$$
\underset{\sim}{\stackrel{\text { irr }}{\varepsilon}}=\underset{\sim}{\stackrel{v}{\varepsilon}}+\underset{\sim}{\varepsilon}
$$

Assuming that the density of the solid part of the samples did not change during the thermal treatment, the velocity of the porosity reduction can be described by (Equation 12):

$$
\dot{\mathrm{f}}=(1-\mathrm{f}) \operatorname{tr} \underset{\sim}{\stackrel{\cdot}{\varepsilon}}
$$

Equations 4, 7, 8, 9, 10, 11 and 12 constitute the system (Equations 13) to be resolved for obtaining the full description of the evolution of the variables of the model in each point of the mechanical system: 


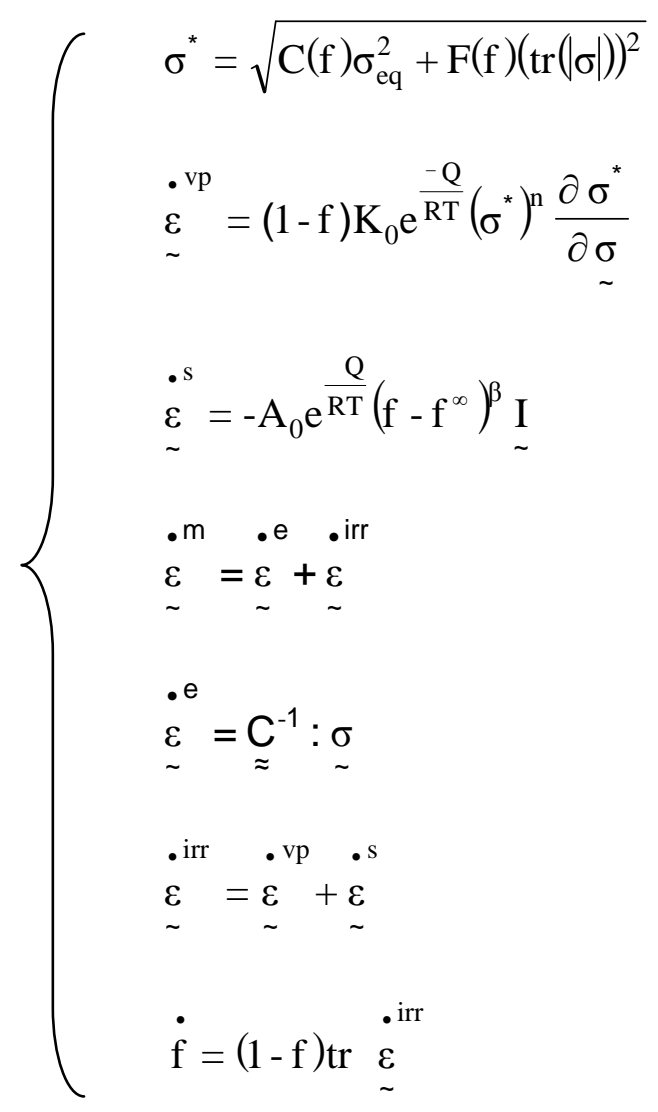

4.2.4. Identification of the materials' parameters for modelling.

The 2D and 3D microstructural investigations have shown the presence of a grain size gradient along with a porosity gradient in the $\mathrm{z}$ direction of our samples, the latter being a consequence of the former. Clearly, a porosity gradient leading to a differential in the sintering kinetics appears to be a good candidate for the driving force for the observed deformation/curvature of our sintered samples. To test this hypothesis, we have modelled the deformation occurring during the sintering of the samples in these terms; the numerical simulation is based on the software "ZéBuLoN", in which the above general system of Equation 13 has been implemented to describe the thermo-mechanical behaviour of the system. The major goal of this study was to adapt the experimental tape casting method to proton conducting BCY-based ceramics. We did not aim at fine-tuning and optimizing all the parameters that have been incorporated in the modelling, rather, by setting the mechanical parameters to reasonable values drawn from the literature, and measuring quantitatively by image analysis the evolution of the surface fraction of pores in the $\mathrm{z}$ direction of crosssections of samples during sintering, we attempted to validate our hypothesis about the gradient of porosity as the major driving force for deformation, in order to correct our shaping process accordingly. Table 2 describes the set of parameters used for the Finite Element modelling. 


\begin{tabular}{llll}
\hline TABLE 2. Parameters set for finite element modelling. & & \\
\hline Parameters & & Co-sintered Electrolyte & Anode \\
\hline Young's modulus & $\mathrm{E} / \mathrm{MPa}$ & $154.103[11]$ & $154.103[11]$ \\
Poisson's coefficient & $v$ & 0.30 & 0.30 \\
Norton's law pre-exponential factor & $\mathrm{K}_{\mathrm{o}}$ & $45563[12]$ & $45563[12]$ \\
Norton's law exponent coefficient & $\mathrm{n}$ & $1.1[12]$ & $1.1[12]$ \\
Activation Energy for the Norton's law & $\mathrm{Q} / \mathrm{kJ} \mathrm{mol}^{-1}$ & 336 & 336 \\
Pre-exponential factor for the sintering & $\mathrm{A}_{\mathrm{o}}$ & 2.106 & 2.106 \\
deformation velocity & $\mathrm{C}(\mathrm{f})$ & 1.0 & 1.0 \\
Function which depends on the porosity & $\mathrm{F}(\mathrm{f})$ & 0 & 0 \\
Function which depends on the porosity & & & 1.2 \\
Sintering deformation velocity exponent & $\beta$ & 1.2 & 0.34 \\
coefficient & & 0.19 & 0.59 \\
Final porosity & $\mathrm{f}^{\infty}$ & 0.50 & \\
Initial porosity & $\mathrm{f}_{\mathrm{o}}$ & &
\end{tabular}

In particular, the elastic properties of the solid phase in the electrolyte (BCY10) and in the anode $(\mathrm{BCY} 10+\mathrm{NiO})$ have been assumed to be the same since it appeared that a full calculation would have been difficult and anyway would have shown a second order influence on the deformation compared to the porosity gradient. In addition, this should not affect the phenomenological description of the deformation that is our primarily objective. Fig. 12 gives the comparison between the experimental quantification of the surface fraction of porosity after interrupted sintering treatments and the corresponding theoretical values given by the model; considering the uncertainties relative to the porosity measurements, it is seen that there is a reasonable agreement between the experimental values and the model, which is hence sufficiently validated to describe the deformation behaviour of our samples during sintering.

\subsubsection{Finite Element modelling.}

\subsubsection{Influence of a porosity gradient: First, we have investigated the influence of a} porosity gradient introduced in the electrolyte green layer. Each layer has been numerically divided into 4 sub-layers, which were attributed the same material parameters but different values of the surface fraction of pores. Two calculations were carried out, the first one taking into account a porosity gradient in the electrolyte, and the second one with no porosity gradient. In both cases the porosity in the anode was considered homogeneous. $\mathrm{f}_{\mathrm{o}}$ and $\mathrm{f}^{\infty}$ are respectively the surface fraction of pores at the onset of the sintering (green tapes) and at the 
end of the sintering (sintered tapes). The initial gradient of porosity is defined by the values taken by $\mathrm{f}_{\mathrm{o}}$ within the electrolyte 4 sub-layers (see Tables 3 and 4).

TABLE 3. Numerical parameters for porosity for an Anode/Electrolyte bi-layer with a porosity gradient.

\begin{tabular}{lllll}
\hline Numerical layer & Material layer & $\mathrm{f}_{\mathrm{o}}$ (initial) & $\mathrm{f}^{\infty}(\mathrm{final})$ & $\mathrm{f}_{\mathrm{o}}-\mathrm{f}^{\infty}$ \\
\hline L8 & Electrolyte & 0.52 & 0.16 & 0.36 \\
L7 & Electrolyte & 0.51 & 0.18 & 0.33 \\
L6 & Electrolyte & 0.49 & 0.20 & 0.29 \\
L5 & Electrolyte & 0.48 & 0.22 & 0.26 \\
L4 & Anode & 0.59 & 0.34 & 0.25 \\
L3 & Anode & 0.59 & 0.34 & 0.25 \\
L2 & Anode & 0.59 & 0.34 & 0.25 \\
L1 & Anode & 0.59 & 0.34 & 0.25 \\
\hline
\end{tabular}

TABLE 4. Numerical parameters for porosity for an Anode/Electrolyte bi-layer without porosity gradient.

\begin{tabular}{lllll}
\hline Numerical layer & Material layer & $\mathrm{f}_{\mathrm{o}}$ (initial) & $\mathrm{f}^{\infty}$ (final) & $\mathrm{f}_{\mathrm{o}}-\mathrm{f}^{\infty}$ \\
\hline L8 & Electrolyte & 0.50 & 0.19 & 0.31 \\
L7 & Electrolyte & 0.50 & 0.19 & 0.31 \\
L6 & Electrolyte & 0.50 & 0.19 & 0.31 \\
L5 & Electrolyte & 0.50 & 0.19 & 0.31 \\
L4 & Anode & 0.59 & 0.34 & 0.25 \\
L3 & Anode & 0.59 & 0.34 & 0.25 \\
L2 & Anode & 0.59 & 0.34 & 0.25 \\
L1 & Anode & 0.59 & 0.34 & 0.25 \\
\hline
\end{tabular}

The results of the calculations are given in Fig. 13 and Fig. 14. It is seen that for the AnodeElectrolyte bi-layer without porosity gradient within the electrolyte, the deformation is very small and is the consequence of a limited differential shrinkage between the two layers during the sintering (Fig. 13). When a gradient of porosity is introduced within the electrolyte, the sintering leads to a large deformation (Fig. 14), confirming the role of this gradient as the major driving force for non-planar deformation acting when the materials shrink. The nonplanar deformation originating from the porosity gradient within the electrolyte, and hence from the sedimentation of the largest particles in the green tapes, is associated with a strong 
scaling effect (see Fig. 15), which could be a major problem for the fabrication of larger diameter cells.

4.2.5.2. Influence of sintering kinetic: In the above calculations, the sintering behaviour for the two layers was assumed to be identical. However, it would be reasonable to assume that the sintering kinetics for the anode (NiO-BCY10) and for the electrolyte (BCY10) are different since the materials are different, which consequently should result in a specific deformation due to this differential. To detect this effect, we have arbitrarily chosen to vary the constant $A_{o}$ (Table 2) in equation [8] from $A_{o}=4.106$ for the electrolyte to $A_{o}=2.106$ for the anode, with no porosity gradient. The evolution of the porosity during sintering was then plotted, and it is seen that the velocity of the porosity evolution is maximum at the beginning of the sintering for both layers (Fig. 16), diminishing thereafter until the end of the thermal treatment where the residual deformation is very small (Fig. 17). The electrolyte sinters more rapidly than the anode at the beginning of the thermal treatment, whereas at the end the anode catches up. This evolution of the curvature of a bi-layer that we have modelled is consistent with in-situ observations made by Ravi and Green [13] on tape cast Alumina layers. It is attributed to a difference of initial density in the different layers.

\subsubsection{Combination of a porosity gradient and the sintering kinetic-3D calculation: The} calculated deformation obtained when an experimental gradient of porosity is incorporated in the numerical model was smaller than the experimental deformation observed on our samples. As already indicated, it is assumed that the non-optimized materials parameters should lead to a second order influence on the total deformation; on the other hand, we have also shown that the model presented above (Eq. [13]) displays a fairly consistent behaviour and trend with those of the real samples. Since 3D morphology instabilities were experimentally observed on sintered bi-layers (oscillations of the disks edges), it is suspected that a $2 \mathrm{D}$ calculation based on the 2D axi-symmetrical hypothesis does not allow us to describe fully all the mechanisms at work during sintering. Therefore a full 3D calculation has been carried out in an attempt to simulate the amplitude of the deformation observed on samples with a diameter of $10 \mathrm{~mm}$. The vertical deformation and displacement of the meshes have been blocked on one side of the sample to orientate the curvature. In that case, the curvature of the sample has significantly increased (Fig. 18) and appeared to be in a good agreement with the experimental deformation, in direction and amplitude. The stress maps at different moments of the sintering (not shown here) indicate that the level of stress increases sharply up to a 
maximum at the onset of the thermal treatment because of the kinetics of the sintering, then decreases until full relaxation of stresses occurs at the end of the sintering. This calculation showed that the amplitude of the deformation is better explained if a combined effect of a porosity gradient and a differential in the sintering kinetics is taken into account. In addition, owing to the high level of stress and deformation at the onset of the sintering, it also gave evidence that the use of a load on top of the samples in order to keep them planar during the thermal treatment should systematically be detrimental to the sample integrity, either by inducing a high level of stress by blocking the deformation, or by giving rise to residual stresses if relaxation by creep is not fully completed. In either cases fracture should occur in the edge vicinity, which is exactly what we have observed experimentally in our samples.

\subsubsection{Conclusion on the modelling.}

The initial porosity gradient may explain the curvature of the membranes after sintering. A phenomenological model has been constructed in which deformation of the specimen is correlated to the evolution of the porosity. This model couples the viscoplastic deformation rate and the sintering deformation rate with thermal activation and porosity before and after thermal treatment processing.

The parameter identification remains to be fine-tuned experimentally and theoretically, but we obtained values in accordance with the literature, which is sufficiently relevant to point out the importance of the porosity gradient and hence the instability of the slurry that gave rise to the sedimentation of the largest particles within the green tapes.

We observed that the porosity gradient due to the sedimentation of the largest particles in the green tapes was responsible for the final curvature of the specimen. We also confirmed that the phenomenon was scale dependent since the curvature of the membranes was larger at higher values of their diameter.

The calculations showed that the curvature varied during the process according to the sintering kinetics of the different materials. Therefore the largest curvature occurred just after the onset of sintering. This phenomenon has already been reported on alumina membranes with a porosity gradient.

Finally, the 3D calculation showed that the samples could experience a higher curvature at the end of an unconstrained treatment; although applying a load on the samples during the process would help to keep them planar, it would, however, lead to residual stresses that give rise to fracture as we observed during our experiments. 


\section{Conclusions}

Tape casting proved to be an efficient shaping process when applied to SOFC or PCFC devices. Whereas water-based tape casting can be applied easily to YSZ-based SOFC, it appeared to be impracticable for BCY10-based PCFCs because of a strong interaction of BCY10 with water. Organic tape casting has been developed specifically for BCY10; planar symmetrical Anode/Electrolyte half-cells have been obtained, but single Anode/Electrolyte bi-layers cannot be sintered without becoming strongly warped. 2D and 3D microstructural investigations (BSE-SEM and X-ray computed microtomography) coupled with a phenomenological model, have confirmed that a gradient of porosity in the green tapes acted as the driving force for curvature formation. The Finite Element numerical simulation has also demonstrated that sintering the bi-layer under a mechanical load to counterbalance the deformation in the $\mathrm{z}$ direction was not viable. Rather, it seems that the solution to the problem is to be found in a slurry formulation which avoids the sedimentation of the larger grains. Therefore, it is concluded that the process should use a narrow distribution grain size with a more careful fine-tuning of the dispersant amount in the slurry.

\section{Aknowledgments}

The authors are grateful to Jacques Besson, David Ryckelynck and Dominique Jeulin (MinesParisTech) for stimulating discussions on mechanical modelling and simulation. The authors are also grateful for having been allowed to carry out experiments at the ESRF and thank the ID19 beamline staff. This work was partly supported by a grant from the French "Agence Nationale de la Recherche" ANR (Project TECTONIC), by the "Groupement des Écoles des Mines" (GEM). 
Fig. 1 Sintering treatment of a single SOFC cell

Fig. 2 Fracture cross-section of a single SOFC cell obtained via tape-casting and co-sintering (SEM)

Fig. 3 SEM on a fracture cross-section of the Electrolyte/Cathode interface of a cell where the cathode was deposited by organic tape casting

Fig. 4 X-Ray diffraction pattern of the as-calcined BCY10 powder

Fig. 5 SEM micrograph of a BCY10 particle

Fig. $6 \mathrm{pH}$ of protonic ceramic powder suspensions in water (in the ratio of 1:10) for different $\mathrm{BaCeO}_{3}$ and $\mathrm{SrCeO}_{3}$ based perovskites

Fig. 7 Thermal treatment for PCFC half-cells sintering

Fig. 8 SE-SEM observation of a fracture surface cross-section of a planar symmetrical halfcell

Fig. 9 BSE-SEM observation of a fracture cross-section of the Anode/Electrolyte interface

Fig. 10 Tomography cross-section of an anode after removal of the organic compounds (350 ${ }^{\circ} \mathrm{C}-1$ hour); largest ceramic particles are seen at the bottom (left)

Fig. 11 Tomography cross-section of an anode layer after sintering is completed $\left(1450{ }^{\circ} \mathrm{C}-\right.$ 24 hours); the largest ceramic particles are again seen at the bottom (left) of the layer and the porosity is not homogeneous in the thickness of the layer

Fig. 12 Comparison of experimental and numerical porosity as a function of the thermal annealing duration

Fig. 13 Finite Elements modelling of the curvature of an Anode/Electrolyte bi-layer without any porosity gradient 
Fig. 14 Finite Elements modelling of the curvature of an Anode/Electrolyte bi-layer with a porosity gradient within the electrolyte layer

Fig. 15 Scale effect on the curvature (sample diameter: 5 and $20 \mathrm{~mm}$ )

Fig. 16 Evolution of the sintering velocity for both anode and electrolyte materials

Fig. 17 Influence of the sintering kinetics on the curvature during sintering

Fig. 18 3D Finite Elements modelling of the curvature after sintering at $1450{ }^{\circ} \mathrm{C}$ for 24 hours, with the combined effect of a porosity gradient and the sintering kinetics 


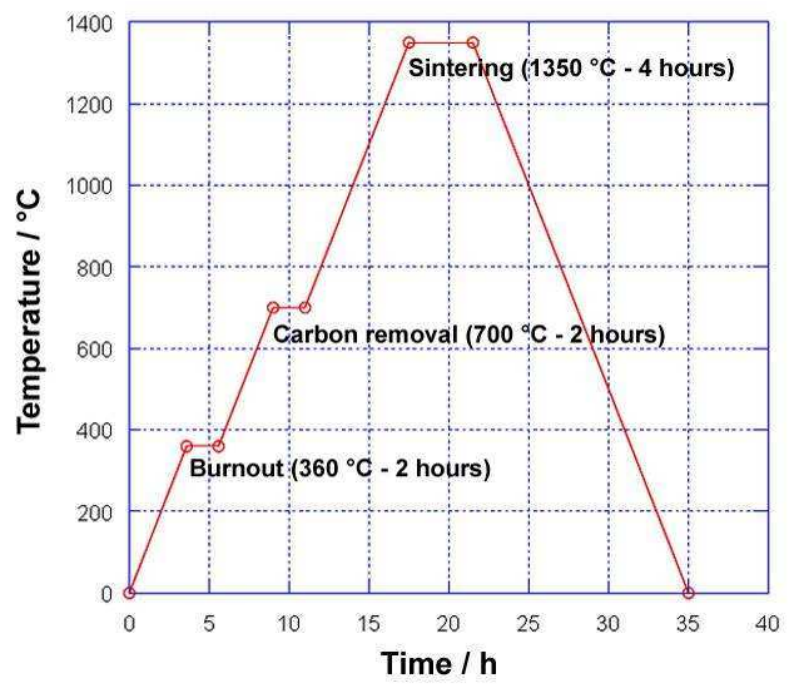

Fig. 1 


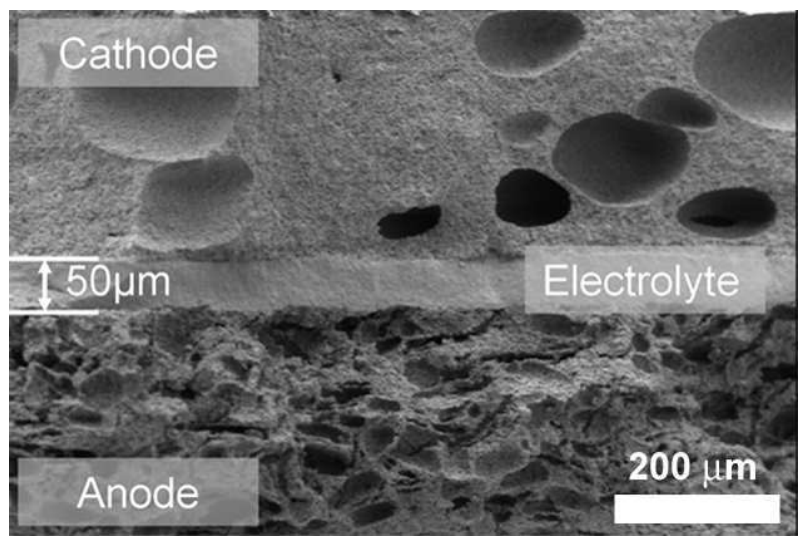

Fig. 2 


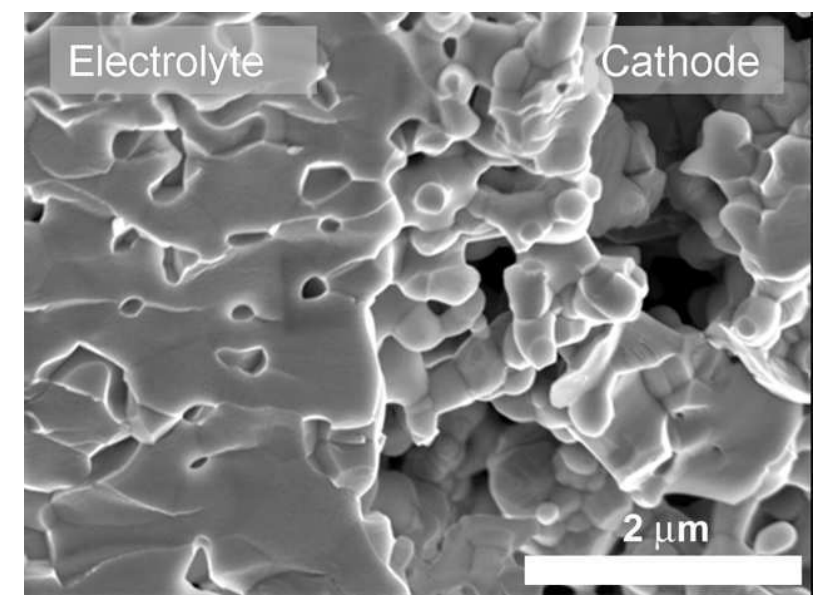

Fig. 3 


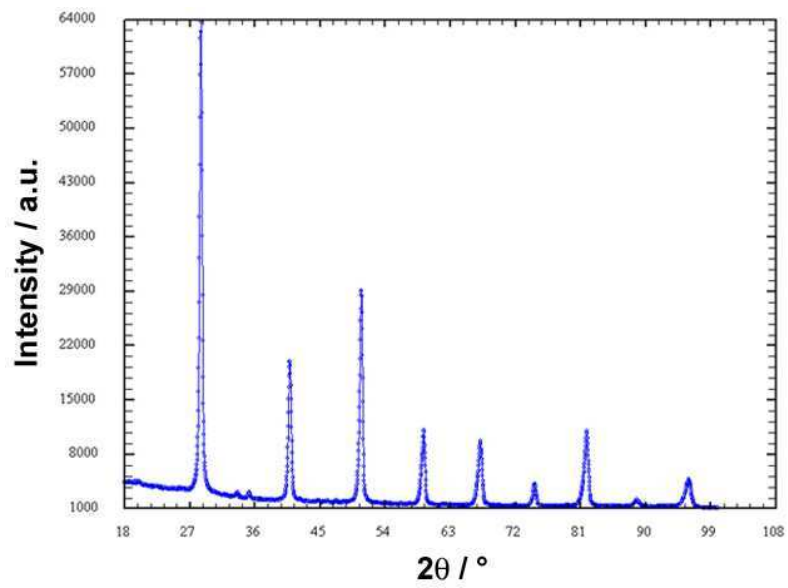

Fig. 4 


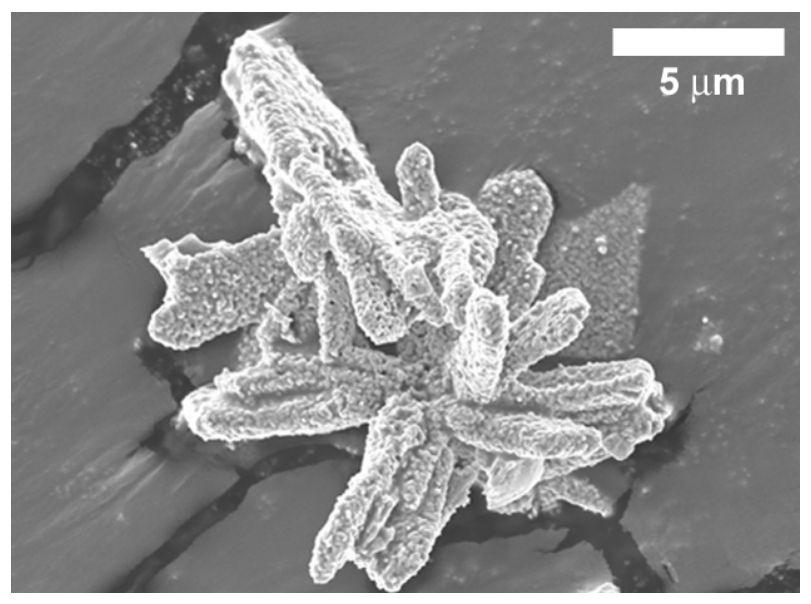

Fig. 5 


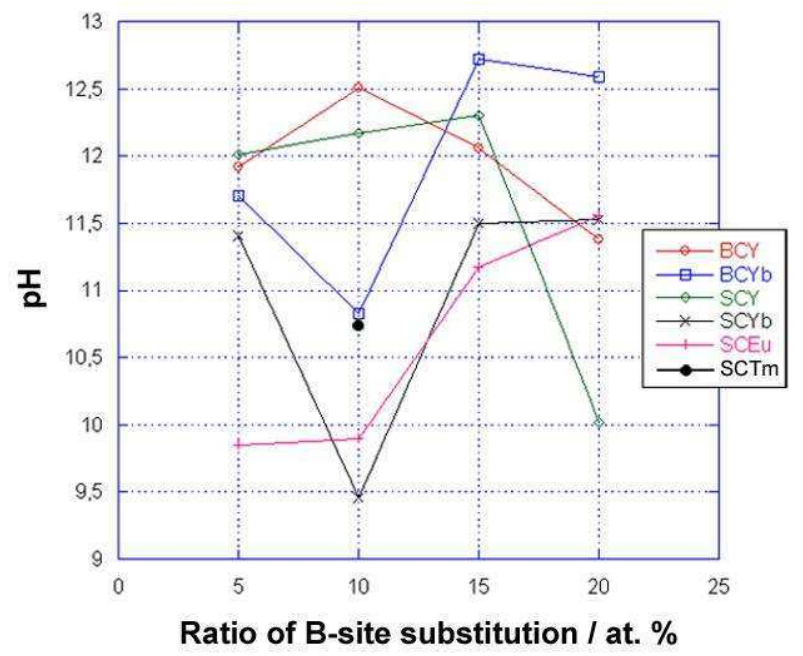

Fig. 6 


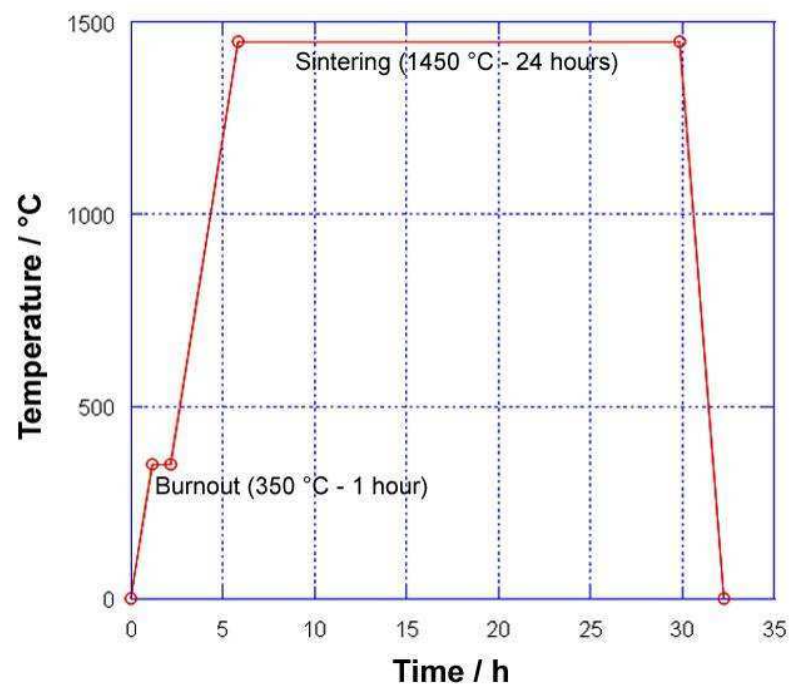

Fig. 7 


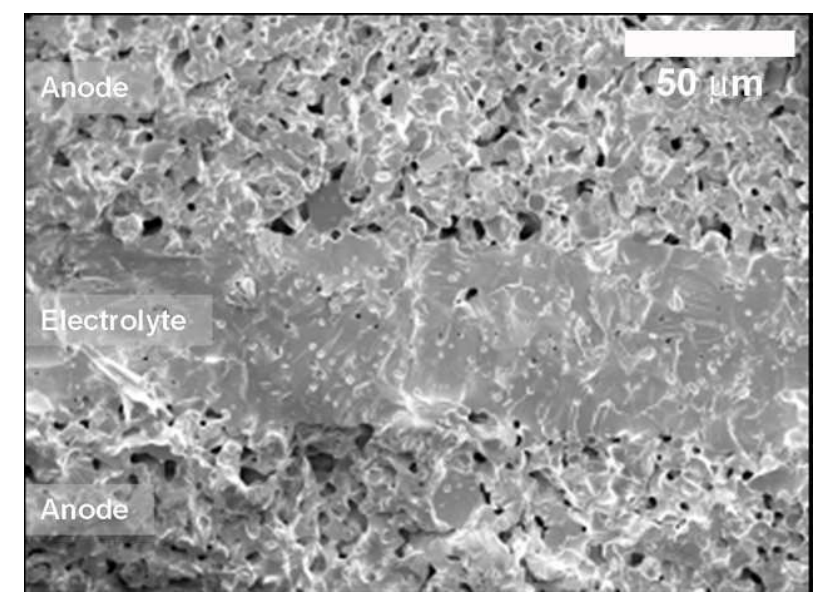

Fig. 8 


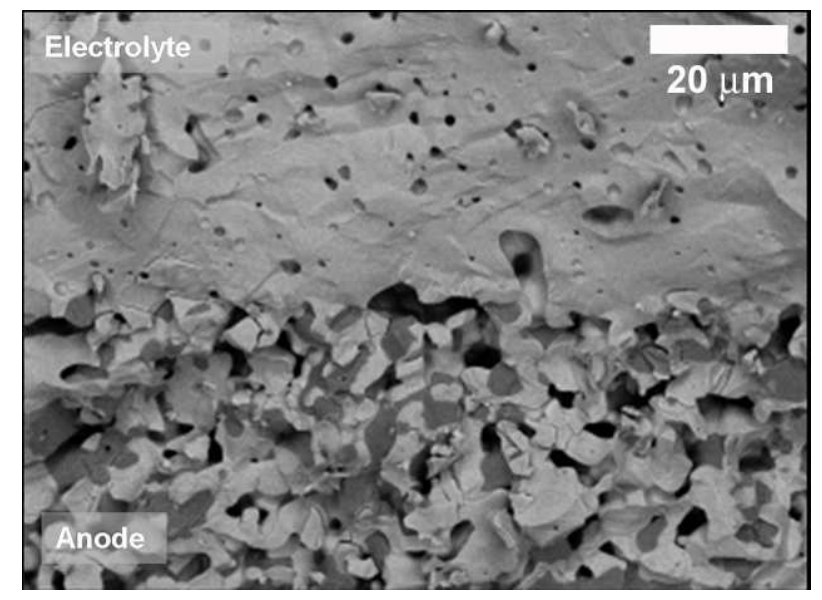

Fig. 9 


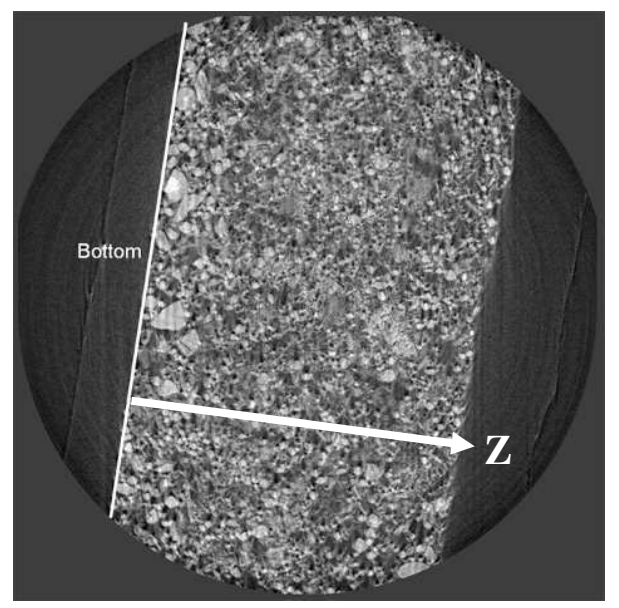

Fig. 10 


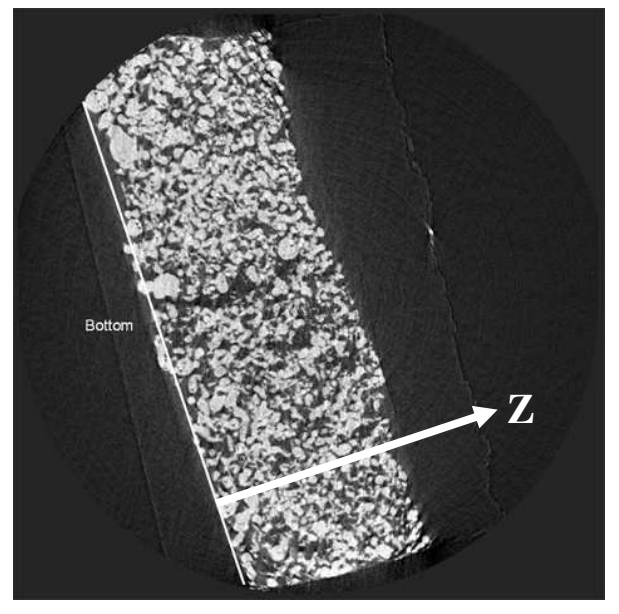

Fig. 11 


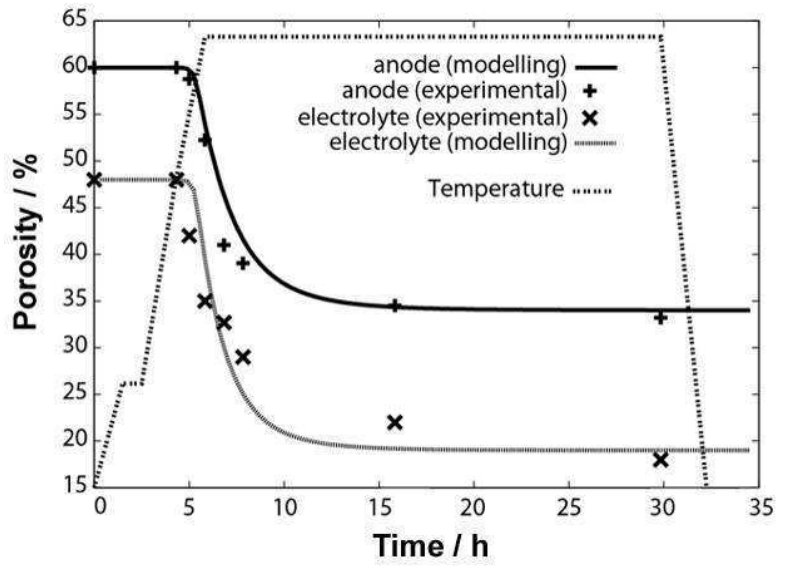

Fig. 12 


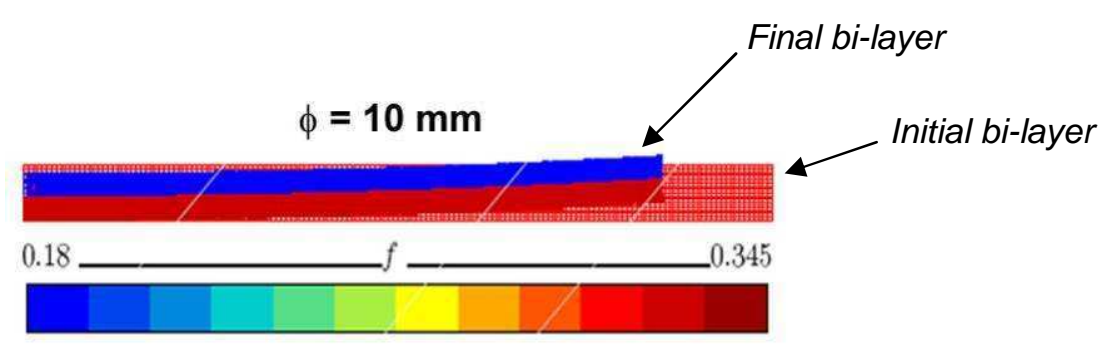

Fig. 13 


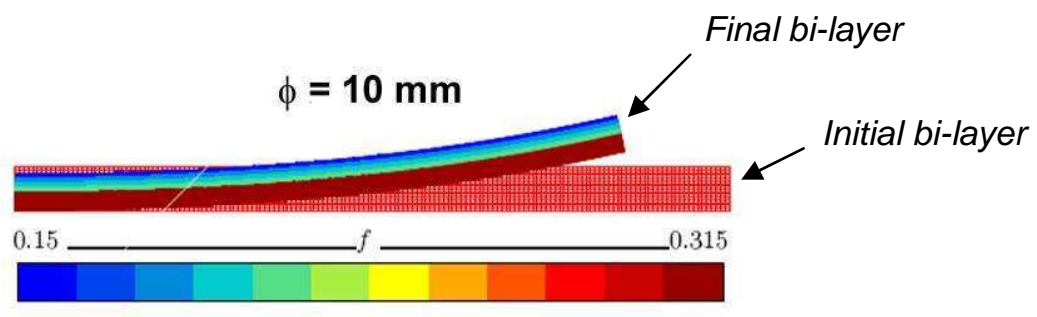

Fig. 14 


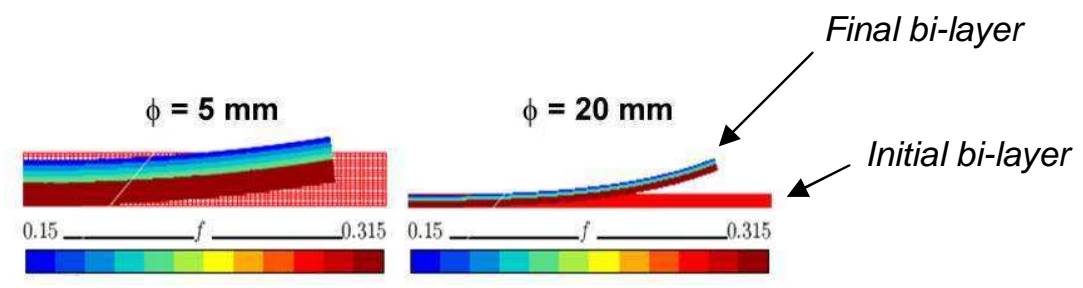

Fig. 15 


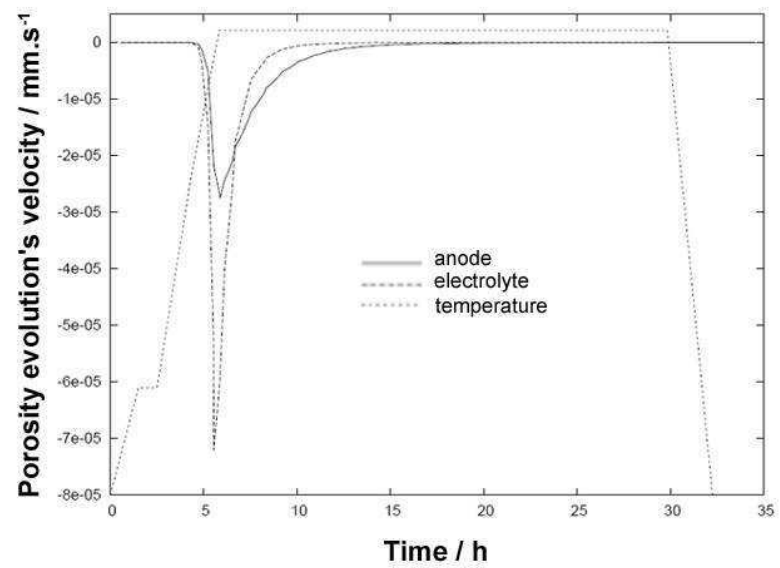

Fig. 16 


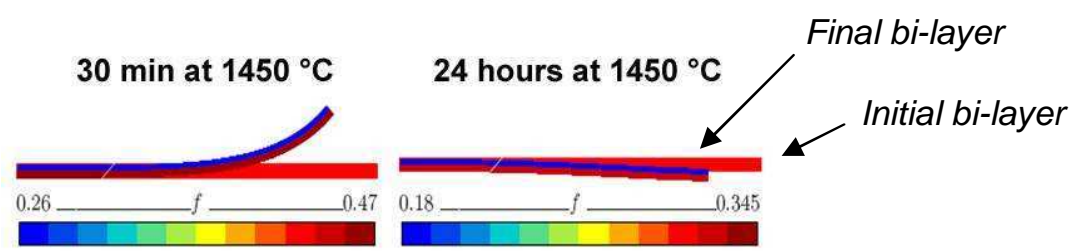

Fig. 17 


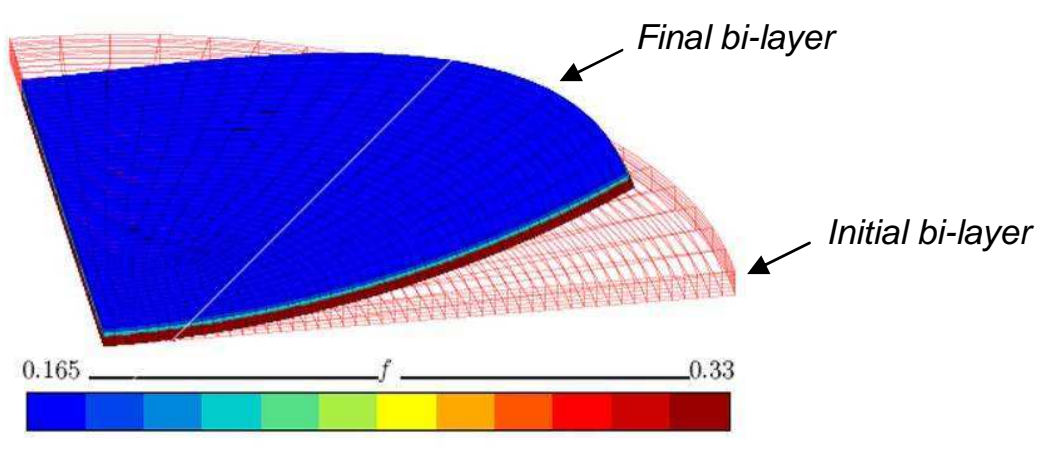

Fig. 18 


\section{References}

$1 \quad$ Moreno R (1992) J Amer Ceram Soc 71:1521

$2 \quad$ Moreno R (1998) J Amer Ceram Soc 71:1647

3 Grosjean A (2004), Etude et réalisation par coulage en bande et co-frittage de cellules de pile à combustible à oxides solides, Evry

4 Timoshenko SP, Gere JM (1991) Mechanics of Mater, Chapman and Hall

5 Grosjean A, Sanséau O, Radmilovic V, Thorel A (2006) Solid State Ionics 177:1977

6 Almeida de Oliveira AP, Hafsaoui J, Hochepied JF, Berger MH, Thorel A (2007) J Eur Ceram Soc 27:3577

7 Costa R, Hafsaoui J, Grosjean A, Berger MH, Jeulin D, Thorel A (2008) J Eur Ceram Soc, Accepted for publication

8 Chen F, Toft-Sørensen O, Meng G, Peng D (1997) J Mater Chem 7:481

9 Guan J, Dorris SE, Balachandran U, Liu M (1998) J Electrochem Soc 145:1780

10 Zhong Z (2007) Solid State Ionics, 178:213

11 Yamanaka S, Fujikane M, Hamaguchi T, Muta H, Oyama T, Matsuda T, Kobayashi SI, Kurosaki K (2003) J Alloys and Comp 359:109

12 Goretta KC, Park ET, Guan J, Balachandran U, Dorris SE, Routbort JL (1998) Solid States Ionics 111:295

13 Ravi D, Green DJ (2006) J Eur Ceram Soc 26:17 